\title{
Development, Testing of Construct Validity and Reliability of Pakistan Senior Citizens Quality of Life- Measurement Tool (PSCQL-MT)
}

\author{
Hamid Mahmood ${ }^{1, \text { * }, \text { Ammara Waqar }}{ }^{2}$, Syed Amir Gilani ${ }^{1}$, Muhammad Yaqoob ${ }^{1}$, \\ Ejaz Mahmood Ahmad Qureshi ${ }^{1}$, Saleem Rana ${ }^{1}$, Asif Hanif ${ }^{1}$, Awais Gohar ${ }^{3}$ \\ ${ }^{1}$ University Institute of Public Health, The University of Lahore, Lahore, Pakistan \\ ${ }^{2}$ Quality Enhancement Cell, Al-Aleem Medical College, Lahore, Pakistan \\ ${ }^{3}$ Primary and Secondary Health Department, Government of the Punjab, Lahore, Pakistan
}

\section{Email address:}

drhamidmahmood373@gmail.com (H. Mahmood), ammarahamid24@gmail.com (A. Waqar), profgilani@gmail.com (S. A. Gilani), yaqoobchich@yahoo.com (M. Yaqoob),ejaz_qureshi@hotmail.com (E. M. $\Lambda$. Qureshi), saleem.uiph@gmail.com (S. Rana), asif.hanif@ahs.uol.edu.pk (A. Hanif),dr.awais.gohar@gmail.com (A. Gohar)

${ }^{*}$ Corresponding author

\section{To cite this article:}

Hamid Mahmood, Ammara Waqar, Syed Amir Gilani, Muhammad Yaqoob, Ejaz Mahmood Ahmad Qureshi, Saleem Rana, Asif Hanif, Awais Gohar. Development, Testing of Construct Validity and Reliability of Pakistan Senior Citizens Quality of Life- Measurement Tool (PSCQLMT). American Journal of Biomedical and Life Sciences. Vol. 8, No. 5, 2020, pp. 156-172. doi: 10.11648/j.ajbls.20200805.15

Received: August 15, 2020; Accepted: August 26, 2020; Published: September 16, 2020

\begin{abstract}
This study has been dedicated to design, create and validate of quality of life (QoL) questionnaire called as (PSCQL-MT). This tool has been developed to measure physical activities, social relationship, cognitive aging, leisure activities and health behaviours. The objective of the study is to Develop, Test of Construct validity and reliability of (PSCQLMT) to measure Quality of Life (QoL) in Senior Citizens of Lahore, Pakistan. Temporal stability was measured using the testretest method. The overall questionnaire score shows an ICC of.86 $(\mathrm{p}<.001)$ with a $95 \%$ confidence interval $(\mathrm{CI})=[.83-.89]$. Analyses by dimensions range from a CCI of.72 $(\mathrm{p}<.001)$ with a $95 \% \mathrm{CI}=[.66-.76]$ for the "other leisure activities" dimension to a CCI of.87 ( $<$.001) with a $95 \% \mathrm{CI}=[.84-.89]$ for the "social activities" dimension. There was no specific valid questionnaire available in Pakistan to evaluate the individual and overall QoL in Pakistan. For this purpose, a reliable and valid construct questionnaire needs to be developed. This study has developed a tool that can measure 5 major variables which determine the QoL It has been concluded from the study that the development of PSCQL-MT questionnaire and valid tool is a great achievement to carry out research on the QoL in the study population.
\end{abstract}

Keywords: PSCQL-MT, Quality of Life (QoL), Measurement Tool, Development, Testing, Reliability, Validity, Senior Citizen, Physical Exercise Activity, Cognitive Aging

\section{Introduction}

Old age is considered to be a negative notation. Which means last period of a normal life which is characterized by the disturbance or partial loss of cognitive executive functions in the senior citizens. Aging is a process which is defined by Maxia "everyone is older than yesterday but younger than tomorrow".

The definition of aging has created two questions about the old age. According to WHO, old age is considered to begin at the age of $65 .[1]$ The recent changes in political decision making about the senior citizens has become of paramount importance. This change in demographic phenomena has led to the investigation on the process of healthy aging. A better understanding of the factors affecting elderly person needs to be evaluated properly in order to make the healthy aging positive. Researchers are working to promote minimum impairment in physical and cognitive functions in the elderly persons.

It is very important before starting the investigation about the factors that influence physical and cognitive abilities should be validated and a proper scale for the measurement of characteristics to be developed based on our previous 
studies. This study has been dedicated to design, create and validate of quality of life (QoL) questionnaire called as "Pakistan Senior Citizens Measurement Tool (PSCQL-MT)". This tool has been developed to measure physical activities, social relationship, cognitive aging, leisure activities and health behaviours.

\section{Theoretical Background}

For the purpose of designing and validation of a measurable tool to check the quality of life $(\mathrm{QoL})$, it is important to design a comprehensive QoL questionnaire. The purpose of this literature review is to find out the factors that influence the QoL and to discuss the various scales that have been used to measure the QoL previously.

There are many factors that influence i.e. physical activities, social relationship, cognitive aging, leisure activities and health behaviours which affect the healthy aging process in many ways.

Gow et al. carried out a research to find out the factors that influence the aging process. He was of the view that physical activities, social relations play a very important role in maintaining the quality of life in the senior citizens. [2]

Blasko et al. carried out the study on the physical activities and cognitive stimulation in the senior citizens. He found out that physical activity leads to cognitive stimulation which improved the QoL in the senior citizens. [3]

Di Marco et al. carried out another study to find out the relationship between the physical activity and social interaction among the senior citizens. He found out that the senior citizens who were physically healthy are more busy in social activities and hence can survive with the healthy aging. [4]

There are various existing methods for the measurement of impact of various factors that influence the QoL. The first objective is to find out the characteristics of every methodology to find out a valid and reliable tool. The second objective is to study the methodological choices which have been carried out for different frameworks for measurement of QoL.

There are two types of researches which have been carried out:

1. Observational: in which the factors effecting the QoL are being measured only

2. The experimental: in which not only the influence is measured but pharmaceutical and non-pharmaceutical interventions has been made to calculate their effect.

Our study is based on experimental interventional program to find out the effect of physical exercise activities and their effect on the physical and cognitive wellbeing in the elderly persons.

Albinet et al. carried out an experimental approach to measure the effect of intervention of physical exercise activity and its relation to cognitive wellbeing in the elderly person. He used the pre-test and post-test methods to find the difference before and after the intervention. [5]

Mortimer et al. carried out another interventional study to find out the relationship of physical activities and cognitive decline in the senior citizens. He found out that the physical activity does not only improve the physical ability to perform activities of daily living (ADL) but also improve cognitive improvement to perform instrumental activities of daily living (IADL). [6]

It is also important to note that the interventional study is very costly in terms of time and money as they need several months of follow-up by professional qualified personals.

For the purpose of discussing various scales, different variables have been used to measure the QoL.

The quality of life (QoL) measurement tools can be divided into three categories:

1. The questionnaires which have been develop to measure QoL in the elderly population. Lawton \& Brody develop a questionnaire to assess the QoL in the elderly persons.[7] Wright et al. develop a questionnaire about the special weight measurement in the children. [8]

2. A questionnaire was developed to measure single specific type of activities. Booth et al. developed a questionnaire to measure the specific physical activity in the elderly. [9] Schinka et al. developed a single specific intellectual measuring activity tool to measure the physical activities in the study population. [10]

3. The researchers in the last decade tried to develop questionnaire beyond single specific factor and tried to develop lifestyle questionnaire. Jopp \& Hertzog develop a general questionnaire which contains physical, social and emotional components about the QoL of elderly persons. This questionnaire contains 12 items about the QoL of the adult population. The same group developed an 82 items questionnaire which was further sub divided into 11 sub categories. These categories can be measured from moderate to good. This needs a lot of time and money to complete the research through this questionnaire. Finally, it has been noted that a questionnaire does not have proper questions regarding the information or key health issues about the senior citizens' QoL. [11]

The researchers were of the view that a more detailed questionnaire is needed to measure the QoL in the population which is based on physical activities, social relationship, cognitive aging, leisure activities and health behaviours.

In spite of the availability of different questionnaires, Kesse-Guyot et al. tried to develop a new questionnaire which was not scientifically approved due to important limitations. The main limitation was 1). It was designed for a specific population. 2). It was specific for a particular activity that cannot measure the overall QoL. 3). The questionnaire was very long. [12]

From the above stated reasons, it is important to develop a new questionnaire which should not only be comprehensive to measure the various QoL dimensions of the elderly population. The new measurement tool has been given in the name Pakistan Senior Citizens Measurement Tool (PSCQL-MT). 


\section{Development of PSCQL-MT}

The first step in development of PSCQL-MT was to identify different variables that affect the health of elderly population. There are five main dimensions:

1. physical

2. mental and/or cognitive health: cognitive

3. social

4. physical and

5. other leisure activities

Small eta al. emphasis that the cognitive health is of utter most importance in the aging population [13] Wang et al. were of the view that the social interaction are very important for the healthy aging in the elderly persons. [14] Steinmo et al. were of the view that the regular physical exercise activities are important for healthy aging in the elderly. [15]

MacDonald et al. emphasizes the importance of leisure activities for health living in the questionnaire for measurement of QoL in the elderly persons. All these factors need to be included in the preparation of questionnaire for the measurement of health living in the elderly person. [16]

The next step was the development of new tool which have variables to be included in the questionnaire. Various studies have been carried out mostly of single specific objective providing a sample tool to measure a single factor.

The study will be carried out to check the validity of PSCQL-MT.

Development, Testing of Construct validity and reliability of Pakistan Senior Citizens Quality of Life - Measurement Tool (PSCQL-MT) to measure Quality of Life (QoL) in Senior Citizens of Lahore, Pakistan

\section{Objective of the Study}

The objective of the study is:

1. To Develop, Test of Construct validity and reliability of Pakistan Senior Citizens Quality of Life - Measurement Tool (PSCQL-MT) to measure Quality of Life (QoL) in Senior Citizens of Lahore, Pakistan

\section{Method}

A five step design has been developed to construct PSCQL-MT to measure Quality of Life (QoL).

These five steps are given below in Figure 1

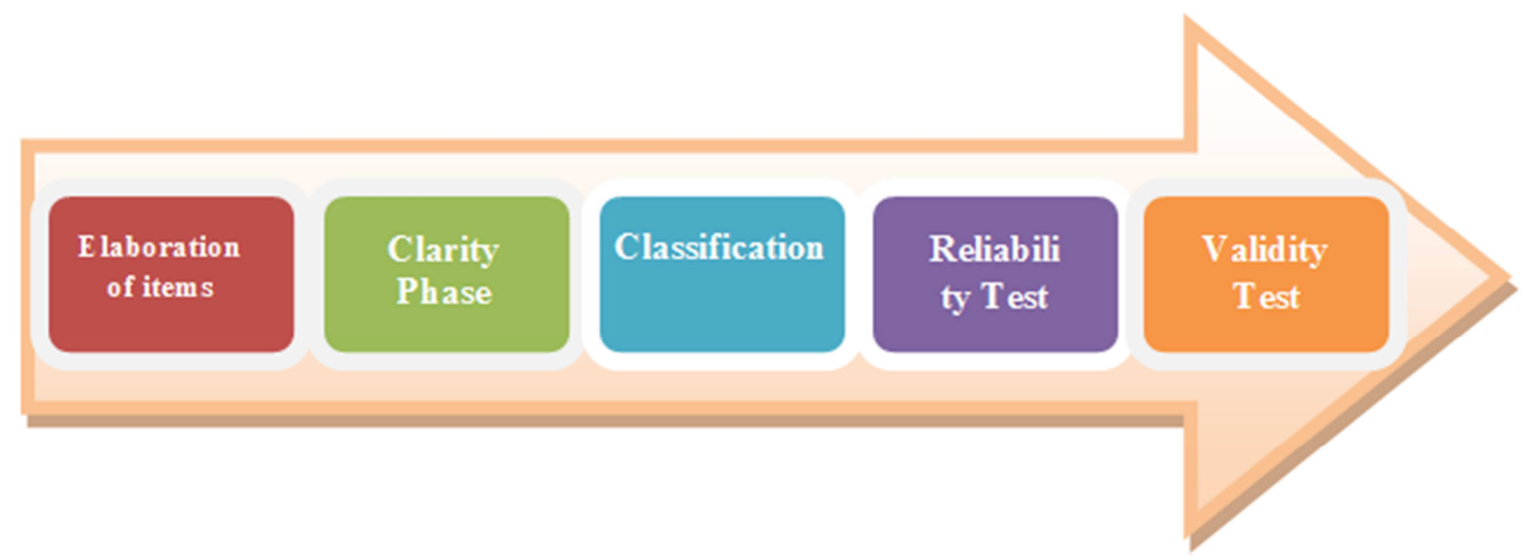

Figure 1. Phases leading from design to validation of the PSCQL-MT.

\subsection{Elaboration of Different Variables in the Questionnaire}

The questionnaire has been prepared to measure the QoL in the elderly persons using five categories i.e. physical, cognitive, social, other leisure activities and other health behaviours. These five domains have been put on five point Likert scale to measure PSCQL-MT questionnaire.

The participants have been chosen and have been made familiar about the filling process of the questionnaire. This strategy has brought pace and comfort to complete the questionnaire. Once the entire questionnaire has been completed, we recruited four separate samples for the validation of PSCQL-MT questionnaire.

\subsection{Participants}

The entire questionnaire was filled by 44 participants, including 26 men and 18 women. The 44 participants in this sample 1 were between ages 21 to $80(M=49.5 ; S D=19.4)$ and had an average of 12 years of study, which is equivalent to a "Higher Secondary" level. The inclusion criteria for this study were the persons of both genders have age between 2180 years (see Figure 2).

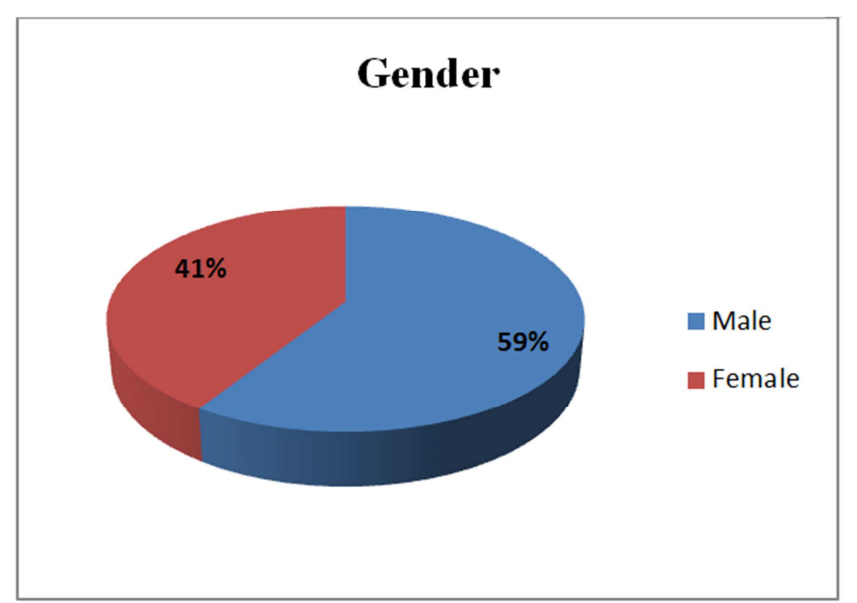



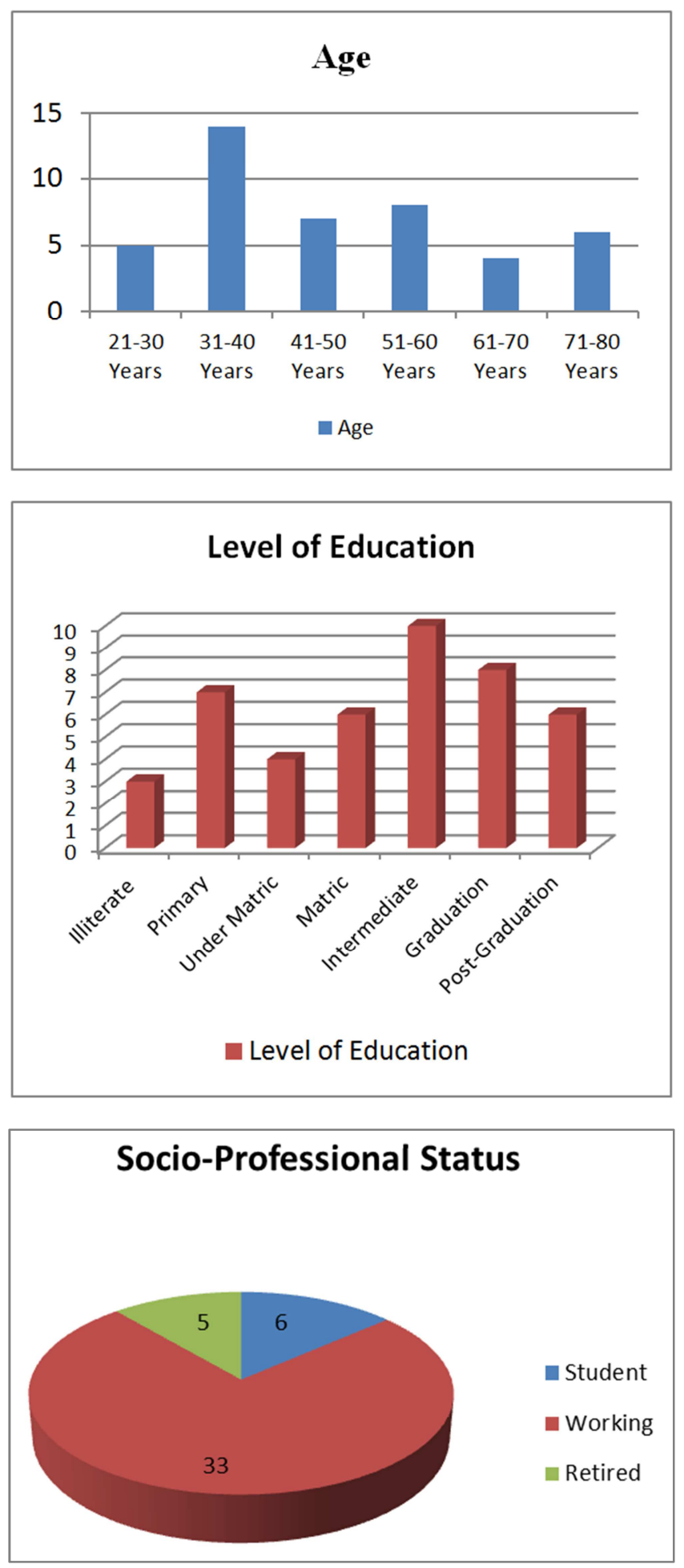

Figure 2. Socio-demographic characteristics of sample 1.

Study Design: Quasi experimental Design

Settings: This study has been conducted in District Lahore, Pakistan.

Duration of Study: Three Months from November 2019 to January, 2020.

Sampling Technique: The data has been collected from senior citizens, health care givers, Old Homes Administrators, Social Welfare Organizations, Hospitals, Non-Government
Organizations, Old People Homes and community at large. Sample Selection

Inclusion Criteria: All persons who have age of 21 years and above, male or female are included in the study.

Exclusion Criteria: All Persons (Male/Female) having age less than 21 years has excluded from the study.

Study Tools: Questionnaire (PSCQL-MT)

Sample size $(n)=44(M=26, F=18)$

Mean: 59.5

$S D: 19.4$

\subsection{Clarity Phase}

The purpose of this phase was to verify the comprehensibility of each variable among the index population.

\subsection{Classification Phase}

The objective of classification phase is to classify each item in the questionnaire according to the lifestyle dimension of the elderly persons. This included physical activities, cognitive and social behaviours, leisure activities and health behaviours.

\subsection{Construct Reliability of the Test}

The reliability of a tool/questionnaire is concerned with the consistency of the measurement. It means, it verifies that the tool measure correctly what it is supposed to measure. The reliability of the PSCQL-MT is estimated using Cronbach alpha which is the measure of internal consistency at one hand and re test the aims to verify time stability on the other hand.

\subsection{Construct of Validity Tests}

In this step, the psychometric property of PSCQL-MT was measured to check the reliability and scientific validity of closely related variables. Validity is the measurement of an instrument what, is supposed to measure and what it measure. Through this test the evaluation of test hypothesis is done and a collaboration measure is used to evaluate the construct validity of questionnaire.

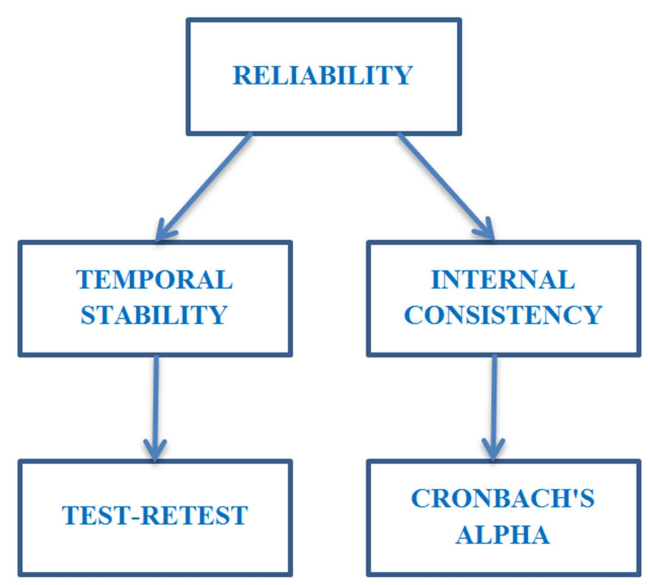

Figure 3. Steps in assessing the psychometric qualities of the PSCQL-MT. 


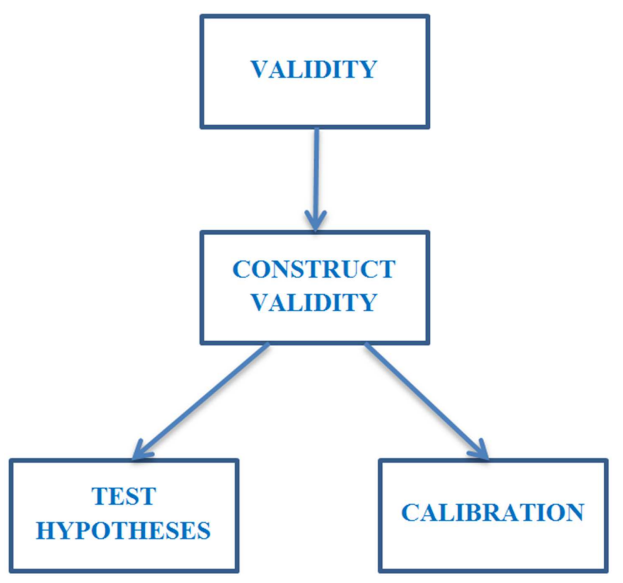

Figure 4. Steps in assessing the psychometric qualities of the PSCQL-MT.

\section{Measuring Tool}

\subsection{PSCQL-MT}

The PSCQL-MT has been designed to evaluate the five important variables that measure the QoL of study population. The analysis of each variable has provided us the information about the QoL in the study population.

\subsection{The Modifiable Activity Questionnaire (MAQ)}

In the study the relationship between the physical exercise activity and the cognitive executive functions has been measured. This study has been carried out for a period of one month for filling filing up of the questionnaire by the study population. In order to make any modification in the questionnaire MAQ will be developed to exclude or include any variable in the tool to measure the QoL in the study population. Which need modification to assess different variable in the best possible way?

\section{Data collection and Data Analysis}

The data of the filled questionnaire has been recorded. The corrected data has been placed in the SPSS version 25. The reliability and construct validity of the questionnaire has been tested at Cronbach's alpha.

\subsection{Reliability of Data Collection and Analysis}

Testing of the reliability is two stages process. In a first step, Cronbach's alpha coefficient is used to measure the internal consistency of the overall scores of all variables defining the QoL in the study population. In the second step, intra-class coefficiency (ICC) has been used to assess the reliability of questionnaire score obtained in the test and re-test.

\subsection{Processing of Valid Data}

Two steps have been used to measure construct validity of the questionnaire. In the first step Pearson correlation has been used to compare the result of physical variable obtained through PSCQL-MT questionnaire and QAM questionnaire.
In the second step, ANOVA and Pearson correlation has been used to relate PSCQL-MT results to test the hypothesis. ANOVA tested test hypothesis were used to check the relationship of the different variables.

\section{Results}

\subsection{Clarity, Classification, Relevance and Completeness of PSCQL-MT Items}

\subsubsection{Clarity}

After analysis of the responses from Sample 1, all items scored between 4 and 4.78 out of 5 (Avg=4.56; SD=0.2), allowing the next phase to proceed without rewriting or deleting any items. This step showed good comprehensibility of the formulation of the PSCQL-MT items by the target population.

\subsubsection{Classification}

The ANOVAs and post-hoc tests conducted to identify the dominant category of each item during the classification phase carried out by sample 2, reveal that two items have indistinguishable scores for several dimensions (see Figure 5). Since it was unclear in which main QoL component to categorize these items. We decided to delete these two items. This deletion of items was done in order to promote a rigorous classification of items by dimension.
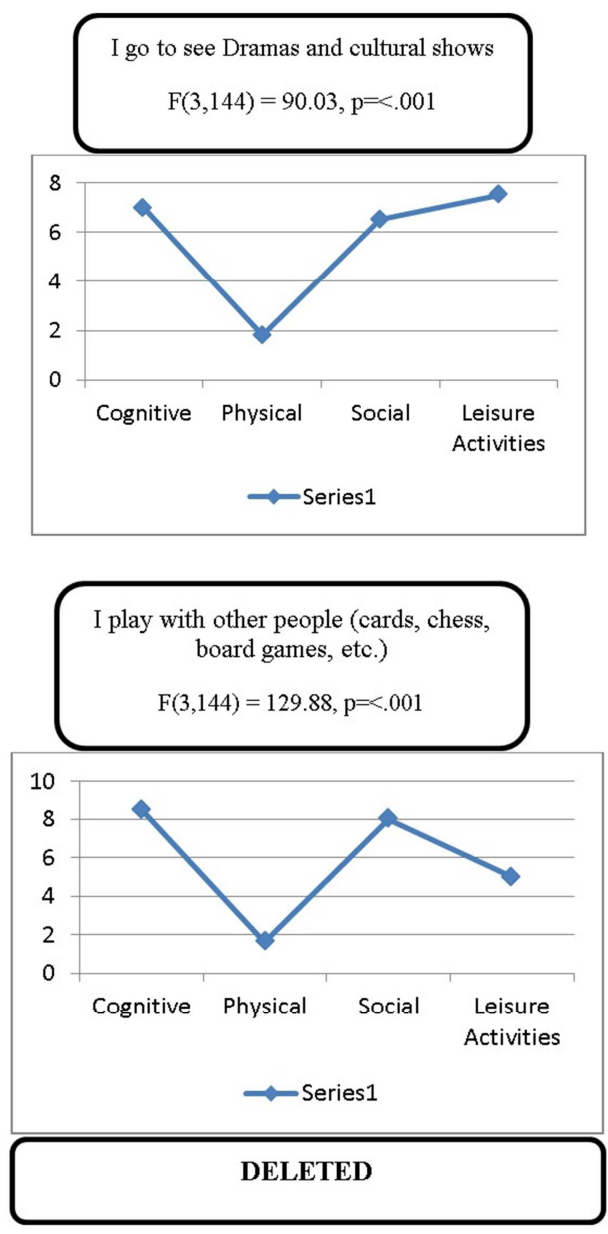

Figure 5. Items deleted as a result of undefined categorization during the classification phase. 
As a result of this external categorization, the PSCQL-MT has 43 items divided into 5 dimensions, as follows namely:

1) Cognitive activities ( 7 items), 2) Social activities (9 items), 3) Physical activities (9 items), 4) Other leisure activities (8 items), 5) Dimension including sleep, food and tobacco consumption (10 items).

\subsubsection{Relevance of Items}

The relevance of the items was assessed by checking for extreme marginal distributions of responses to the items. Thus, it is estimated that if an item obtains a rate greater than $97 \%$ for the same response (e.g., "Every day or almost every day"), then the relevance of the item is highly questionable.[17] Indeed, under these conditions, the item does not allow for differentiation between participants and is generally suppressed. Based on the responses provided by sample 3, all PSCQL-MT items scored below 97\%, thus meeting the inclusion criteria.

\subsubsection{Completeness of PSCQL-MT Items}

As said before, one of the main objectives when designing the PSCQL-MT was to offer a tool that was as complete as possible. In order to check whether this objective had been achieved, the question "Have you carried out any other activities (not previously mentioned) in the last 12 months? this was asked at the end of the questionnaire.

Out of 44 participants, only 4 (or $1.34 \%$ ) answered "yes" to this question. They were then asked for details of the activities in question. The following activities were mentioned as missing: "music" $(\mathrm{N}=3)$, "health care" $(\mathrm{N}=3)$, "training" $\quad(\mathrm{N}=3), \quad$ "business management" $\quad(\mathrm{N}=2)$, "photography" ( $\mathrm{N}=2)$, "Drawing" $(\mathrm{N}=1)$. Since these items were mentioned no more than three times (i.e., $0.28 \%$ of the sample) and appear to correspond to rare activities, we decided not to add them to the PSCQL-MT. While not exhaustive, the PSCQL-MT nevertheless appears to provide a comprehensive overview of Qol.

\subsection{Reliability of the PSCQL-MT}

\subsubsection{The Temporal Stability of the PSCQL-MT}

Temporal stability was measured using the test-retest method $(\mathrm{N}=44)$. The overall questionnaire score shows an ICC of.86 (p <.001) with a 95\% confidence interval $(\mathrm{CI})=[.83-.89]$. Analyses by dimensions range from a CCI of.72 $(\mathrm{p}<.001)$ with a $95 \% \mathrm{CI}=[.66-.76]$ for the "other leisure activities" dimension to a CCI of.87 $(\mathrm{p}<.001)$ with a $95 \%$ $\mathrm{CI}=[.84-.89]$ for the "social activities" dimension. The PSCQL-MT therefore shows good temporal stability.

\subsubsection{Internal Consistency}

Internal consistency was measured by calculating Cronbach's alpha coefficients for the overall score and each dimension. The overall Cronbach's alpha score is good (.72). Scores by dimension are generally moderate (.54 for the dimension sleep, diet and tobacco consumption) to very good (.75 for physical activity). However, "cognitive" and "other leisure time activities" showed poorer internal consistency (.46 and.31 respectively).

The results of these two reliability tests are detailed in Table 1.

Table 1. Summary table of PSCQL-MT reliability results.

\begin{tabular}{|c|c|c|c|c|}
\hline \multirow{3}{*}{ Global and Dimensional Analysis } & \multicolumn{3}{|c|}{ Temporal Stability } & \multirow{3}{*}{$\begin{array}{l}\text { Internal Consistency } \\
\text { Cronbach's Alpha }\end{array}$} \\
\hline & \multirow{2}{*}{ ICC } & \multicolumn{2}{|c|}{ 95\% Confidence Interval } & \\
\hline & & Lower bound & Upper bound & \\
\hline Quality of Life (QoL) & .860 & .825 & .888 & .72 \\
\hline Cognitive activities & .804 & .764 & .838 & .46 \\
\hline Physical activity & .793 & .730 & .840 & .75 \\
\hline Social activities & .871 & .843 & .894 & .59 \\
\hline Other leisure activities & .717 & .662 & .763 & .31 \\
\hline Other health behaviors & .866 & .837 & .889 & .55 \\
\hline
\end{tabular}

* ICC $=$ Inter Class Correlation.

Interpretation: $\geq .8$ - Good; $\geq .7$ - Satisfactory; $\geq .6$ - Medium; $\geq .5$ - Low; $\leq .5$ Very low $[18,19]$.

\subsection{The Validity of the PSCQL-MT Construct}

Two methods were used to measure the construct validity of the PSCQL-MT. The first method consists in relating the results found in the literature to those found using PSCQL-MT. Many test hypotheses from the literature were confirmed through the responses of the participants in the sample (see Table 2).

Table 2. Summary table of validated test hypotheses.

\begin{tabular}{|c|c|c|c|}
\hline Variable 1 & Variable 2 & Hypothesis Test & Statistical processing \\
\hline Physical Activities & Age & Participation in physical activity decreases with age & $r=-.28, p<.001$ \\
\hline BMI & Physical Activities & BMI is negatively correlated with physical activity & $r=-.24, p<.001$ \\
\hline Cognitive Activities & Year of schooling & Participation in cognitive activities is positively related to years of schooling & $r=.11, p<.001$ \\
\hline Social Activities & Age & Participation in social activities is inversely related to age & $r=-.51, p<.001$ \\
\hline Social Activities & Gender & $\begin{array}{l}\text { The frequency of participation in social activities is not significantly } \\
\text { different between men and women }\end{array}$ & $F(1,44)=.19$, n.s \\
\hline Religious Activities & Gender & Women more likely to participate in religious activities than men & $F(1,44)=4.40, p<.05$ \\
\hline
\end{tabular}




\begin{tabular}{|c|c|c|c|}
\hline Variable 1 & Variable 2 & Hypothesis Test & Statistical processing \\
\hline Health Behaviour & Professional status & Pensioner said they had more healthy behaviors than the rest of the sample & $F(1,44)=32.73, p<.001$ \\
\hline Smoking & Educational level & A negative relationship between education and smoking & $r=-.14, p<.001$ \\
\hline Food + Smoking & Cigarette & Smokers more often report having bad eating habits & $F(1,924)=27.35, p<.001$ \\
\hline Food & Professional status & Students more often reported having poor eating habits than other statuses & $F(1,44)=34.63, p<.001$ \\
\hline Sleep & Gender & Women have more frequent reports of sleep disorders than men & $F(1,44)=4.87, p<.001$ \\
\hline Lifestyle & Educational level & $\begin{array}{l}\text { People with higher education have a richer lifestyle than people with less } \\
\text { education }\end{array}$ & $r=.08, p<.01$ \\
\hline
\end{tabular}

The second method consists of comparing the results obtained by the physical dimension of the PSCQL-MT with the results obtained by the QAM. The relationship between these two tools shows a significant correlation $(\mathrm{r}=.528$, $\mathrm{p}=<.01$ ). Thus, Pearson's correlation between the "physical" dimension of the PSCQL-MT and the scientifically validated questionnaire "QAM" seems to confirm the construct validity of this dimension of the PSCQL-MT.

\subsection{Presentation of the Final Version of the PSCQL-MT}

The PSCQL-MT measures the frequency of participation in 43 types of activities and behaviours over the past 3 months (see Annex-1). At the end of the classification phase, the PSCQL-MT increased from 46 to 43 items, broken down as follows:

Cognitive activities" includes 7 items that involve one or more brain functions, such as attention, memory and reasoning:

1. I visit museums, exhibitions, etc.

2. I play individual thinking games (puzzles, quizzes, etc.) regardless of the medium (paper, computer, mobile phone or game console).

3. I read "scientific" articles, newspapers, novels, etc. for private or professional purposes.

4. I write by hand (letters, note-taking, diaries, etc.).

5. I write on a computer (e-mail, report, minutes, etc.)

6. I watch news, documentaries, current affairs magazines, cultural programmes, etc.

7. I do creative activities (I draw, paint, sculpt, sew, knit, etc.).

Social activities" are 9 items and include regular social activities (e.g., spending time with friends, visiting family) as well as the types of social interactions that have become more common since the development of new technologies (e.g., conversations via SMS, Skype, social networks):

1. I go to the movies or see entertaining shows (circus, one-man show, concerts, etc.)

2. I go out in the city, to restaurants, cafes... with other people.

3. I take part in voluntary actions and/or get involved in a club or association (sports, school, neighbourhood, political party, etc.).

4. I attend religious events

5. I share time with my family (family meals, visits to my children/parents/grandparents, etc.).

6. I use social networks (Facebook, Twitter, WhatsApp, etc.)

7. I use self-help networks offered on the Internet (couchsurfing, carpooling sites, car or apartment rentals between individuals, etc.).

8. I have oral conversations, whether face-to-face, by phone, via Skype, webcam, etc.

9. I have written conversations, whether by SMS, chat, email, etc.

Physical activities" includes 9 items and takes into account competitive sports practices, leisure time physical activities, as well as physical activities of daily life.

1. I practice sports, fitness or leisure activities that require me to be physically active for at least 10 minutes at a time: A low level of physical effort. My heart rate increases slightly. I can talk without being out of breath (walking quietly, playing petanque, fishing, etc.).

2. I practice sports, fitness or leisure activities requiring at least 10 minutes in a row: Moderate physical effort. My heart rate and breathing are increasing. I am out of breath but can continue to talk (water gymnastics, cycling or fun swimming, brisk walking, etc.).

3. I practice sports, fitness or leisure activities requiring: An intense physical effort. My heart rate and breathing increase sharply. Talking becomes difficult or even impossible (jogging, tennis, sports swimming, mountain biking, etc.).

4. In the course of my work and/or my daily tasks, I perform for at least 10 minutes at a time: Low physical effort. My heart rate is slightly higher than usual. I can talk without being out of breath (walking, etc.).

5. In the course of my work and/or my daily tasks, I perform for at least 10 minutes in a row: Moderate physical effort. My heart rate and breathing increase. I am out of breath but can continue to talk (lifting light loads, climbing stairs, etc.).

6. In the course of my work and/or my daily tasks, I perform for at least 10 minutes in a row: Intense physical effort. My heart rate and breathing increase significantly. Talking becomes difficult or even impossible (lifting heavy loads, digging up the garden, etc.).

7. I encourage an active life (taking the stairs, walking, cycling etc.) to go to work, shopping, etc.

8. I maintain my home (do-it-yourself, gardening, cleaning, etc.)

9. I walk more than one hour a day (cumulative time)

The "other leisure activities" dimension presents 8 items and concerns activities practiced mainly for relaxation, pleasure and/or personal enrichment:

1. I read people magazines, sports newspapers, comic strips, etc. for pleasure or relaxation.

2. I watch on television (or on my computer) 
entertainment programs, sports events, movies, series, videos, etc.

3. I stroll, meditate, practice relaxing activities, etc.

4. I take care of my collections (of stamps, figurines, cars, etc.), my flowers, file my photos, etc.

5. I listen to music

6. I use the internet for purposes other than chatting, games and videos (searching or buying on the internet, creating a website or blog, etc.).

7. I visit new places (cities, villages, countries, etc.)

8. I cook for pleasure

The "other health behaviours" dimension, including sleep, eating and smoking, includes 10 items:

1. I smoke cigarettes

2. I use tobacco in other forms (pipe, cigar, chicha, etc.)

3. I drink beverages

4. I eat fruit and vegetables daily.

5. I eat fatty foods (fried foods, deli meats, burgers, etc.)

6. I eat potato chips and other appetizers (crackers, peanuts, pistachios, etc.).

7. I eat pastries and other sweets (sweets, candies, cookies, chocolates, etc.)

8. I eat between meals

9. During the week or when I work, I feel rested when I get up (feeling like I've had enough sleep).

10. On weekends and holidays, I feel rested when I get up (feeling like I've had enough sleep).

The calculation methods are presented below:

1. Score by items according to the answers*:

2. Every day or almost every day $=5$

3. A few times a week $=4$

4. A few times a month $=3$

5. A few times a year $=2$

6. Never $=1$

*Except for items 9, 10, 11, 13, 14, 15, 16 for which the scores are reversed (every day or almost every day $=1$, a few times a week=2, etc.).

1. Score by dimensions:

2. Score $=$ average of items per dimension

3. Cognitive activities: 1 to 8

4. Physical activities: 26 to 34 .

5. Social activities: 35 to 43 .

6. Other leisure activities: 19 to 25 .

7. Health behaviours (eating, smoking and sleeping): 9-18.

8. Overall score:

Score $=$ average of dimensions

In the continuation of this study all of these behaviours related to eating, sleeping and tobacco consumption will be included under the heading of "health behaviours".

\section{Discussion}

The objective of the study was to develop and validate a questionnaire for the purpose of assessment of QoL in the study population. There was no specific valid questionnaire available in Pakistan to evaluate the individual and overall QoL in in Pakistan. For this purpose, a reliable and valid construct questionnaire which should cover all the demographic, socio-economic and cultural variable needs to be develop.

The main purpose of this study was to develop a valid comprehensive tool which can answer the questions raised in this study. This questionnaire will provide the data and the result to this questionnaire will provide us a way to prevent cognitive decline and improve physical health. The PSCQLMT questionnaire has been tested for its psychometric qualities which have been tested with Cronbach's Alpha. The internal consistency of PSCQL-MT questionnaire/tool has proved to be satisfactory. The analysis of test/re-test has showed an excellent temporal stability of the PSCQL-MT questionnaire/tool through overall scores and individual scores or variables. The comparison of validity tools and modifiable activity questionnaire result tested is satisfactory construct validity.

This study has shown several successes. It has developed a tool that can measure 5 major variables which determine the QoL that is physical activities, social relationship, cognitive aging, leisure activities and health behaviours.

According to our knowledge the PSCQL-MT is known to be first validity questionnaire which simultaneously allows the evaluation of five different variables in Pakistan. More importantly the physical activity measurement shown the comparison for the physical health activity globally. [20]

Kim et al. carried out a research on the impact of physical activity on the health of the aging population. He found out that the physical activities plays an important role in improving the QoL in citizens. This is in-persistence with the results of our study which has been using the tested and retested questionnaire and tool of PSCQL-MT questionnaire. [21]

Lampinen et al. carried out another study regarding the leisure time activities and physical activities in the senior citizens. He found out that the physical activities and leisure activities have great impact in maintaining the QoL in the senior citizens. Again the result of this study is consistent with the previous study which further validated our newly developed PSCQL-MT questionnaire. Our tool contains the usage of new technologies by the senior citizens which play an important role in the wellbeing of aging citizens. [22]

Kuo \& Tang carried out a research on the impact of use of new technologies by the elderly. He found out that the use of Facebook, WhatsApp and other activities of mobile and the computer improve the QoL in the elderly population. Our study through this newly developed PSCQL-MT questionnaire showed the same results which were collected by them. [23]

Brown et al. carried out a study to find out the impact of cognitive activity and the association between Social Activity and Cognitive Performance. He carried out a longitudinal study to find out the result of cognitive activity meditation and its effect on social activity and cognitive performance. He observed that the cognitive activity exercises played a great role in maintaining the physical and mental health in the senior citizens. [24]

The questionnaire was administrated through a small sample to test the psychometric quality of internal 
consistency through Cronbach's alpha. The result showed that the tested/re-tested were almost the same which further confirms and reliability of PSCQL-MT. This study which was carried out on wide range of variables to measure QoL who have different occupation and level of education. The result showed that this questionnaire and tool can be used in the diverse population. The result of these analyses showed significant flexibility regarding the use of this questionnaire and tool which will be an added advantage for research work.

\section{Limitations \& Further Recommendations}

The PSCQL-MT questionnaire was developed to collect the data quickly and easily from the different group of people who are in the age range of $21-80$ years. In order to quickly administrate, several activities under the same domain of social activities, physical activities, leisure time to assess the validity of constructed questionnaire. Due to the reason, the answer provided the information on frequency but lack quantitative details in sub categories of variables. This is due to the reason that the Lickert Scale for each item were used in order to administrate and rapid collection of data. The cognitive activities and other leisure activities obtained low internal consistency scores on the basis of Cronbach's alpha. The low scores of Cronbach's alpha may be due to wide range of activities being used in physical activities and other leisure activities.

It can be said that the development of new questionnaire/tool to assess the lifestyle and QoL in the study population is a great achievement which may open new avenues for research.

\section{Conclusion}

It has been concluded from the study that the development of PSCQL-MT questionnaire and valid tool is a great achievement to carry out research on the QoL in the study population. Development of questionnaire and the tool is not limited to the study of QoL but it will lead to opening of new avenues for the development of different tools for the measurement of different variables for the assessment and evaluation in the study population.

\section{Appendix}

\section{Appendix 1. Questionnaire Pakistan Senior Citizens Measurement Tool (PSCQL-MT)}

The purpose of this questionnaire is to collect data of your activities carried out during the last 12 months.

For each item, you will be asked to rate the frequency of the complete over the past year.

1. I visit museums, exhibitions etc.
a) Almost every day
b) Several times a week
c) A few times a month
d) A few times a year
e) Never

2. I play games in any support (indoor or outdoor)
a) Almost every day
b) Several times a week
c) A few times a month
d) A few times a year
e) Never

3. I read for the purpose of pleasure or relaxation magazines, sports, newspapers
a) Almost every day
b) Several times a week
c) A few times a month
d) A few times a year
e) Never

4. I read for private or business use. (newspapers and TV shows)
a) Almost every day
b) Several times a week 

c) A few times a month
d) A few times a year
e) Never

5. I write by hand (letters, note taking, diary, etc.)
a) Almost every day
b) Several times a week
c) A few times a month
d) A few times a year
e) Never

6. I use computer (email, entertainment etc.)
a) Almost every day
b) Several times a week
c) A few times a month
d) A few times a year
e) Never

7. I watch the TV news, documentaries, news magazines, cultural programs, etc.
a) Almost every day
b) Several times a week
c) A few times a month
d) A few times a year
e) Never

8. I practice creative activities
a) Almost every day
b) Several times a week
c) A few times a month
d) A few times a year
e) Never

9. I smoke cigarettes
a) Almost every day
b) Several times a week
c) A few times a month
d) A few times a year
e) Never

10.I consume tobacco in other forms (pipe, cigar, hookah, etc.)
a) Almost every day
b) Several times a week
c) A few times a month
d) A few times a year
e) Never

11.I drink beverages
a) Almost every day
b) Several times a week
c) A few times a month
d) A few times a year 
e) Never

12.I eat fruit or vegetables daily
a) Almost every day
b) Several times a week
c) A few times a month
d) A few times a year
e) Never

13.I eat fatty foods (fries, sausages, burger, etc.)
a) Almost every day
b) Several times a week
c) A few times a month
d) A few times a year
e) Never

14.I eat chips and other snacks (crackers, peanuts, pistachios, etc.)
a) Almost every day
b) Several times a week
c) A few times a month
d) A few times a year
e) Never

15.I eat pastries and other sweets (candies, sweets, biscuits, chocolates, etc.)
a) Almost every day
b) Several times a week
c) A few times a month
d) A few times a year
e) Never

16.I eat between meals
a) Almost every day
b) Several times a week
c) A few times a month
d) A few times a year
e) Never

17.During the week or when I work, I feel rested while waking up (like I had enough sleep)
a) Almost every day
b) Several times a week
c) A few times a month
d) A few times a year
e) Never

18. On weekends and holidays, I feel rested while waking up (like I had enough sleep)
a) Almost every day
b) Several times a week
c) A few times a month
d) A few times a year
e) Never

19.I watch on TV (or on my computer) entertainment programs, sporting events, movies, series, videos, etc. 

a) Almost every day
b) Several times a week
c) A few times a month
d) A few times a year
e) Never

20.I meditate and practice relaxing activities, etc.
a) Almost every day
b) Several times a week
c) A few times a month
d) A few times a year
e) Never

21.I take care of my collections
a) Almost every day
b) Several times a week
c) A few times a month
d) A few times a year
e) Never

22.I listen to music
a) Almost every day
b) Several times a week
c) A few times a month
d) A few times a year
e) Never

23.I also use the Internet for purposes other than chat, games and videos
a) Almost every day
b) Several times a week
c) A few times a month
d) A few times a year
e) Never

24.I visit new places (cities, towns, countries, etc.)
a) Almost every day
b) Several times a week
c) A few times a month
d) A few times a year
e) Never

25.I cook
a) Almost every day
b) Several times a week
c) A few times a month
d) A few times a year
e) Never

26.I practice for at least 10 minutes at a fitness or recreational activities requiring: A low physical effort. My heart rate increases slightly. I can talk without being out of breath (quiet walking, bowling, fishing etc.)

a) Almost every day 
b) Several times a week

c) A few times a month

d) A few times a year

e) Never

27.I practice for at least 10 minutes at a stretch sports, fitness or recreational activities requiring: A moderate physical effort. My heart rate and my breathing increase. I am short of breath but can keep talking (water aerobics, cycling or swimming fun, fast walking, etc.)
a) Almost every day
b) Several times a week
c) A few times a month
d) A few times a year
e) Never

28.I practice for at least 10 minutes at a stretch sports, fitness or recreational activities requiring: an intense physical effort. My heart rate and my breathing increased sharply. Talking becomes difficult or impossible (jogging, tennis, sports swimming, biking, etc.)
a) Almost every day
b) Several times a week
c) A few times a month
d) A few times a year
e) Never

29. As part of my work and/or my daily tasks, I realize for at least 10 minutes in a row: A low physical effort. My heart rate increases slightly compared to usually. I can talk without being out of breath (vacuuming, walking, etc.)
a) Almost every day
b) Several times a week
c) A few times a month
d) A few times a year
e) Never

30. As part of my work and/or my daily tasks, I realize for at least 10 minutes in a row: Moderate physical effort. My heart rate and my breathing increase. I am short of breath but can keep talking (light lifting, climbing stairs, etc.)
a) Almost every day
b) Several times a week
c) A few times a month
d) A few times a year
e) Never

31.As part of my work and/or my daily tasks, I realize for at least 10 minutes in a row: Intense physical exertion. My heart rate and my breathing increased substantially. Talking becomes difficult or impossible (heavy lifting, digging the garden, etc.)
a) Almost every day
b) Several times a week
c) A few times a month
d) A few times a year
e) Never

32.I favor an active life (take the stairs, to walk, bike...) to go to work, shopping, etc.
a) Almost every day
b) Several times a week
c) A few times a month 
d) A few times a year

e) Never

33.I maintain my accommodation (DIY, gardening, cleaning, etc.)
a) Almost every day
b) Several times a week
c) A few times a month
d) A few times a year
e) Never

34.I walk over an hour a day (cumulative time)
a) Almost every day
b) Several times a week
c) A few times a month
d) A few times a year
e) Never

35.I go to the movie theatre or watch entertaining shows
a) Almost every day
b) Several times a week
c) A few times a month
d) A few times a year
e) Never

36.I go to the restaurant with other people
a) Almost every day
b) Several times a week
c) A few times a month
d) A few times a year
e) Never

37.I participate in voluntary activities
a) Almost every day
b) Several times a week
c) A few times a month
d) A few times a year
e) Never

38.I attend religious events
a) Almost every day
b) Several times a week
c) A few times a month
d) A few times a year
e) Never

39.I share time with my family (family meals, visit my children/parents etc.)
a) Almost every day
b) Several times a week
c) A few times a month
d) A few times a year
e) Never 
40.I use social networks ( Facebook, Twitter, WhatsApp etc.)
a) Almost every day
b) Several times a week
c) A few times a month
d) A few times a year
e) Never

41.I use support networks available on the internet
a) Almost every day
b) Several times a week
c) A few times a month
d) A few times a year
e) Never

42.I have oral conversations, either face to face, by telephone, via Skype, a webcam etc.
a) Almost every day
b) Several times a week
c) A few times a month
d) A few times a year
e) Never

43.I have written conversations, either by SMS, chat, email, etc.
a) Almost every day
b) Several times a week
c) A few times a month
d) A few times a year
e) Never

Methods of calculating PSCQL-MT scores Score by items according to answers:
a) Almost every day $=5$
b) Several times a week $=4$
c) A few times a month=3
d) A few times a year $=2$
e) Never $=1$
Except for items9, 10, 11, 13, 14, 15, 16
For which the scores are reversed (almost every day $=1$, several times a week $=2$, etc.).

Score by dimensions:
a) Score=average of items per dimension
b) Physical Activities $=26$ to 34
c) Cognitive Activities $=1$ to 8
d) Social Activities $=35$ to 43
e) Other Leisure Activities $=19$ to 25
f) Health Behaviours $=9$ to 18
Overall score
Score $=$ average of dimensions

\section{Appendix 2. Pakistan Senior Citizens Measurement Tool (PSCQL-MT) Questionnaire items and dimensions}

Sr. No.
1
2
3
4
5
6
7
8
9
10
11

Dimension

Cognitive activity Cognitive activity Cognitive activity Cognitive activity Cognitive activity Cognitive activity Cognitive activity Cognitive activity Health Behaviour Health Behaviour Health Behaviour
Item

I visit museums, exhibitions etc.

I play games in any support (indoor or outdoor)

I read for the purpose of pleasure or relaxation magazines, sports, newspapers I read for private or business use. (newspapers and TV shows)

I write by hand (letters, note taking, diary, etc.)

I use computer ( email, entertainment etc.)

I watch the TV news, documentaries, news magazines, cultural programs, etc. I practice creative activities

I smoke cigarettes

I consume tobacco in other forms (pipe, cigar, hookah, etc.)

I drink beverages 


$\begin{array}{ll}\text { Sr. No. } & \text { Dimension } \\ 12 & \text { Health Behaviour } \\ 13 & \text { Health Behaviour } \\ 14 & \text { Health Behaviour } \\ 15 & \text { Health Behaviour } \\ 16 & \text { Health Behaviour } \\ 17 & \text { Health Behaviour } \\ 18 & \text { Health Behaviour } \\ 19 & \text { Other Leisure activities } \\ 20 & \text { Other Leisure activities } \\ 21 & \text { Other Leisure activities } \\ 22 & \text { Other Leisure activities } \\ 23 & \text { Other Leisure activities } \\ 24 & \text { Other Leisure activities } \\ 25 & \text { Other Leisure activities }\end{array}$

Physical activity

Physical activity

Physical activity

Physical activity
Item

I eat fruit or vegetables daily

I eat fatty foods (fries, sausages, burger, etc.)

I eat chips and other snacks (crackers, peanuts, pistachios, etc.)

I eat pastries and other sweets (candies, sweets, biscuits, chocolates, etc.)

I eat between meals

During the week or when I work, I feel rested while waking up (like I had enough sleep)

On weekends and holidays, I feel rested while waking up (like I had enough sleep)

I watch on TV (or on my computer) entertainment programs, sporting events, movies, series, videos, etc.

I meditate and practice relaxing activities, etc.

I take care of my collections

I listen to music

I also use the Internet for purposes other than chat, games and videos

I visit new places (cities, towns, countries, etc.)

I cook

I practice for at least 10 minutes at a fitness or recreational activities requiring: A low

physical effort. My heart rate increases slightly. I can talk without being out of breath (quiet walking, bowling, fishing etc.)

I practice for at least 10 minutes at a stretch sports, fitness or recreational activities requiring: A moderate physical effort. My heart rate and my breathing increase. I am short of breath but can keep talking (water aerobics, cycling or swimming fun, fast walking, etc.)

I practice for at least 10 minutes at a stretch sports, fitness or recreational activities requiring: an intense physical effort. My heart rate and my breathing increased sharply. Talking becomes difficult or impossible (jogging, tennis, sports swimming, biking, etc.) As part of my work and/or my daily tasks, I realize for at least 10 minutes in a row: A low physical effort. My heart rate increases slightly compared to usually. I can talk without being out of breath (vacuuming, walking, etc.)

As part of my work and/or my daily tasks, I realize for at least 10 minutes in a row:

Moderate physical effort. My heart rate and my breathing increase. I am short of breath but can keep talking (light lifting, climbing stairs, etc.)

As part of my work and/or my daily tasks, I realize for at least 10 minutes in a row: Intense physical exertion. My heart rate and my breathing increased substantially. Talking becomes difficult or impossible (heavy lifting, digging the garden, etc.) I favor an active life (take the stairs, to walk, bike...) to go to work, shopping, etc. I maintain my accommodation (DIY, gardening, cleaning, etc.)

I walk over an hour a day (cumulative time)

I go to the movie theatre or watch entertaining shows

I go to the restaurant with other people

I participate in voluntary activities

I attend religious events

I share time with my family (family meals, visit my children/parents etc.)

I use social networks (Facebook, Twitter, WhatsApp etc.)

I use support networks available on the internet

I have oral conversations, either face to face, by telephone, via Skype, a webcam etc.

I have written conversations, either by SMS, chat, email, etc.

\section{References}

[1] WHO. (2015). World Report on Aging and Health. Retrieved from http://apps.who.int/iris/bitstream/10665/186469/1/WHO_FW C_ALC_15.01_eng.pdf.

[2] Gow, A. J., Pattie, A., \& Deary, I. J. (2017). Lifecourse Activity Participation From Early, Mid, and Later Adulthood as Determinants of Cognitive Aging: The Lothian Birth Cohort 1921. The Journals of Gerontology Series B: Psychological Sciences and Social Sciences, 72 (1), 25-37. doi: 10.1093/geronb/gbw124.

[3] Blasko, I., Jungwirth, S., Kemmler, G., Weissgram, S., Tragl, K. H., \& Fischer, P. (2014). Leisure time activities and cognitive functioning in middle European population-based study. European Geriatric Medicine, 5 (3), 200-207. doi: 10.1016/j.eurger.2013.09.003. 
[4] Di Marco, L. Y., Marzo, A., Muñoz-Ruiz, M., Ikram, M. A., Kivipelto, M., Ruefenacht, D.,... \& Frangi, A. F. (2014). Modifiable lifestyle factors in dementia: a systematic review of longitudinal observational cohort studies. Journal of Alzheimer's Disease, 42 (1), 119-135. 10.3233/JAD-132225.

[5] Albinet, C. T., Abou-Dest, A., André, N. and Audiffren, M. (2016). Improvement in executive function following a 5month aqua-gym program in the elderly: Role of cardiac vagal control in inhibition performance. Biological Psychology, 115, 69-77. doi: 10.1016/j.biopsycho.2016.01.010.

[6] Mortimer, J. A., Ding, D., Borenstein, A. R., DeCarli, C., Guo, Q., Wu, Y.,... \& Chu, S. (2012). Changes in brain volume and cognition in a randomized trial of exercise and social interaction in a community-based sample of non-demented Chinese elders. Journal of Alzheimer's Disease, 30 (4), $757-$ 766. doi: 10.3233/JAD-2012-120079.

[7] Lawton, M. P., \& Brody, E. M. (1970). Assessment of older people. Nursing Research, 19 (3), 278. doi: 10.1097/00006199-197005000-00029.

[8] Wright, N. D., Groisman-Perelstein, A. E., Wylie-Rosett, J., Vernon, N., Diamantis, P. M., \& Isasi, C. R. (2011). A lifestyle assessment and intervention tool for pediatric weight management: the HABITS questionnaire. Journal of human nutrition and dietetics, 24 (1), 96-100. doi: 10.1111/j.1365277x.2010.01126.x.

[9] Booth, J. E., Schinka, J. A., Brown, L. M., Mortimer, J. A., \& Borenstein, A. R. (2006). Five-Factor Personality Dimensions, Mood States, and Cognitive Performance in Older Adults. Journal of Clinical and Experimental Neuropsychology, 28 (5), 676-683. doi: 10.1080/13803390590954209.

[10] Schinka, J. A., Mcbride, A., Vanderploeg, R. D., Tennyson, K., Borenstein, A. R., \& Mortimer, J. A. (2005). Florida Cognitive Activities Scale: initial development and validation. Journal of the International Neuropsychological Society, 11 (1), 108-116. doi: 10.1017/s1355617705050125.

[11] Jopp, D., \& Hertzog, C. (2010). Assessing adult leisure activities: an extension of a self-report activity questionnaire. Psychological assessment, 22 (1), 108. doi: 10.1037/a0017662.

[12] Kesse-Guyot, E., Andreeva, V. A., Lassale, C., Hercberg, S., \& Galan, P. (2014). Clustering of midlife lifestyle behaviors and subsequent cognitive function: a longitudinal study. American journal of public health, 104 (11), 170-177. doi: 10.2105/AJPH.2014.302121.

[13] Small, B. J., Dixon, R. A., McArdle, J. J., \& Grimm, K. J. (2012). Do changes in lifestyle engagement moderate cognitive decline in normal aging? Evidence from the Victoria Longitudinal Study. Neuropsychology, 26 (2), 144-155. doi: $10.1037 / \mathrm{a} 0026579$
[14] Wang, H. X., Xu, W., \& Pei, J. J. (2012). Leisure activities, cognition and dementia. Biochimica et Biophysica Acta (BBA)-Molecular Basis of Disease, 1822 (3), 482-491. doi: 10.1016/j.bbadis. 2011.09.002.

[15] Steinmo, S., Hagger-Johnson, G., \& Shahab, L. (2014). Bidirectional association between mental health and physical activity in older adults: Whitehall II prospective cohort study. Preventive Medicine, 66, 74-79. doi: 10.1016/j.ypmed.2014.06.005.

[16] MacDonald, R., Kreutz, G., \& Mitchell, L. (2012). Music, health, and wellbeing. Oxford University Press. doi: 10.1093/acprof:oso/9780199586974.001.0001.

[17] Sorman, E., Sundstrom, A., Ronnlund, M., Adolfsson, R., \& Nilsson, L. G. (2014). Leisure Activity in Old Age and Risk of Dementia: A 15-Year Prospective Study. The Journals of Gerontology Series B: Psychological Sciences and Social Sciences, 69 (4), 493-501. doi: 10.1093/geronb/gbt056.

[18] George, D., \& Mallery, M. (2003). Using SPSS for Windows step by step: a simple guide and reference.

[19] Peterson, R. A. (1994). A meta-analysis of Cronbach's coefficient alpha. Journal of consumer research, 21 (2), 381391.

[20] WHO. (2010). Global recommendations for physical activity for health. Retrieved from http://www.who.int/dietphysicalactivity/factsheet_recommend ations/en/.

[21] Kim, S., Popkin, B. M., Siega-Riz, A. M., Haines, P. S., \& Arab, L. (2004). A cross-national comparison of lifestyle between China and the United States, using a comprehensive cross-national measurement tool of the healthfulness of lifestyles: the Lifestyle Index. Preventive medicine, 38 (2), 160-171. doi: 10.1016/j.ypmed.2003.09.028.

[22] Lampinen, P., Heikkinen, R.-L., \& Ruoppila, I. (2000). Changes in Intensity of Physical Exercise as Predictors of Depressive Symptoms among Older Adults: An Eight-Year Follow-Up. Preventive Medicine, 30 (5), 371-380. doi: 10.1006/pmed.2000.0641.

[23] Kuo, T., \& Tang, H. L. (2014). Relationships among personality traits, Facebook usages, and leisure activities-A case of Taiwanese college students. Computers in Human Behavior, 31, 13-19. doi: 10.1016/j.chb.2013.10.019.

[24] Brown CL, Robitaille A, Zelinski EM, Dixon RA, Hofer SM, Piccinin AM. Cognitive Activity Mediates the Association between Social Activity and Cognitive Performance: A Longitudinal Study. Psychol Aging. 2016; 31: 831-846. 10.1037/pag0000134. 